\title{
Rastreamento para câncer de mama e estadiamento
}

\author{
Breast cancer screening and stage at diagnosis
}

É publicado, neste fascículo, o estudo de Martins et al. ${ }^{1}$, "Câncer de mama em Goiânia: análise temporal do estádio clínico do tumor ao diagnóstico". Trata-se de um estudo retrospectivo com o objetivo de avaliar a distribuição dos casos de câncer de mama em diferentes estádios clínicos no período compreendido entre 1989 a 2003. O artigo tem importância prática por ter sido realizado num grande centro de referência para tratamento de pacientes provenientes não só da cidade de Goiânia, mas do interior e de outros Estados - inclusive da região Norte do país.

A inclusão de 3.204 pacientes mostra um número expressivo de casos, porém, por ser um trabalho retrospectivo, houve uma perda aproximada de $54 \%$ das pacientes. Isso porque, em 2008, a incidência estimada para Goiânia foi de 600 casos novos por ano, o que resulta na projeção de cerca de 7.000 ocorrências ${ }^{1}$ no período estudado

Uma das explicações é o fato de terem sido incluídas apenas pacientes catalogadas no Registro de Câncer de Base Populacional (RCBP) de Goiânia, que coleta somente casos com diagnóstico confirmado em mulheres que residem no município de Goiânia há, pelo menos, seis meses antes da data do diagnóstico. Se considerarmos que $30 \%$ das pacientes atendidas em São Paulo e no Rio de Janeiro são provenientes de outros Estados do Brasil, parte significativa dos casos não incluídos eram de pacientes advindas de Estados do norte ou nordeste, ou da zona rural ${ }^{2}$. Possivelmente, essa limitação em obter dados da totalidade das pacientes tratadas levou os autores a esta restrição. Evidentemente, os resultados não refletem a realidade do atendimento médico como um todo, pelo contingente não avaliado de mulheres provenientes de áreas rurais com maior proporção de casos avançados.

O Brasil tem carência de estudos dessa natureza por falta de dados coletados ou imprecisão dos mesmos. A pouca ênfase atribuída à coleta dos mesmos, e a ausência ou dificuldade de padronizar dados e condutas contribuem para que profissionais da área da Saúde não deem tanta importância ao preenchimento dos prontuários e à inclusão de dados significativos de determinada neoplasia.

Os casos de carcinoma ductal e lobular não invasivos corresponderam a apenas 1,6 e $4,2 \%$ de todos os casos, respectivamente, evidenciando um número bem inferior em relação ao dos países que realizam programas de rastreamento mamográfico, nos quais a proporção é próxima de $20 \%{ }^{3}$. Vale ressaltar que, nos últimos cinco anos, há indicadores de que o número

'Professor da Disciplina de Mastologia da Universidade Federal de São Paulo - UNIFESP - São Paulo (SP), Brasil; Diretor do Hospital Pérola Byington - São Paulo (SP), Brasil. 
de casos tenha aumentado ${ }^{2}$, apesar do rastreamento oportunístico nas grandes cidades dos Estados das regiões Sul e Sudeste.

Pela inexistência de um programa nacional de rastreamento populacional, cada localidade vem desenvolvendo ações isoladas que podem beneficiar determinadas populações (rastreamento oportunístico). Apesar de todas essas ações, a cobertura mamográfica, no Estado de Goiás, de apenas $46 \%$ para mulheres com mais de 40 anos está próxima dos dados do Datasus (2003), mas está aquém dos 70\% de cobertura mamográfica necessários para a redução da taxa de mortalidade pela neoplasia. Um dado muito importante foi a observação crescente do número de casos de carcinoma in situ, que se elevou de $0,2 \%$, no primeiro qüinqüênio, e 6,2\%, no terceiro, compatíveis com resultados do rastreamento oportunístico ${ }^{4}$. O Estado de São Paulo é atualmente o que desenvolve mutirões oportunísticos com intuito de reduzir o tempo de espera pelo exame e apresenta os melhores indicadores do Brasil, com cerca de $11 \%$ dos casos de neoplasia em sua forma in situ ${ }^{2}$.

Apesar de o estadiamento TNM preconizado pela União Internacional Contra o Câncer (UICC) ser o mais utilizado em câncer de mama, os Registros de Câncer de Base Populacional seguem as recomendações da International Agency for Research on Cancer (IARC), baseado na extensão da doença, pelo SEER nos Estados Unidos. Estes, entretanto, possuem uma base de dados complementares ampla e incluem informações histopatológicas do tumor primário e linfonodos ${ }^{3}$.

O estadiamento com dados clínicos possui falhas interpretativas e de mensuração em até $30 \%$ dos casos, em razão da imprecisão dos dados. Uma limitação do presente estudo para avaliação temporal foi o comprometimento linfonodal axilar, iniciada pelo RCBP de Goiânia a partir de 1994. Antes dessa data, todos os casos de câncer de mama com metástases linfonodais eram classificados como metastáticos.

O estadiamento da neoplasia nos 15 anos estudados evidenciou que 45,6\% dos casos eram localizados, 19,7\% eram regionais, 10,2\% tinham metástases a distância no momento do diagnóstico e 4,2\% eram tumores in situ. Nessa análise, não havia informação quanto ao estadiamento da doença ao diagnóstico para 20,3\% dos casos.

O estudo evidenciou que o percentual de casos de câncer metastático sofreu redução de $24,1 \%$, no primeiro período, para 6,3\% no último, com diminuição de 17,7\%. Ressalte-se que os casos com comprometimento linfonodal eram classificados como metastáticos até 1994; após esta data, os autores relatam que, com o aprimoramento profissional dos registradores de câncer, esses tumores passaram a ser classificados como regionais, comprometendo a real interpretação dos dados apresentados.

O presente estudo evidenciou que $57 \%$ das pacientes tinham mais de 50 anos de idade e que 15,2\% tinham 40 anos ou menos. Este achado está de acordo com o relatado em outras séries de casos, mostrando que o câncer de mama é mais frequente em mulheres com mais de 50 anos de idade, e que não há dados mais recentes atestando aumento na incidência em mulheres jovens. Não é possível afirmar, pelo presente estudo, que o prognóstico das mais jovens seja pior do que das demais e nem que o aumento do número absoluto de casos (249\%) tenha sido apenas pelo crescimento da população ou por aumento da incidência, podendo também ser atribuído à crescente abrangência do registro de câncer de Goiânia 5 .

Nos últimos dez anos, inúmeros trabalhos internacionais evidenciaram aumento do número de casos de câncer da mama feminina. Entretanto, após a redução do uso de terapia hormonal, observou-se uma redução significativa da incidência na Inglaterra e nos Estados Unidos². O fato de não ter havido, neste estudo, diminuição da incidência da neoplasia mamária nos últimos anos avaliados não pode ser atribuído à continuação do uso de terapia hormonal, por não se dispor de dados mais recentes. Além disso, o rastreamento oportunístico acarreta aumento gradativo na incidência.

Quanto ao estadiamento, os resultados apresentados no presente trabalho diferem daqueles observados em países desenvolvidos. Nos Estados Unidos, os tumores localizados exclusivamente na mama foram mais frequentes, variando de 54 a $72 \%$ dos casos, seguido por tumores com extensão regional (comprometimento axilar), com taxas entre 23 e 38\%. Para os tumores com metástases a distância ao diagnóstico, eles observaram taxas de 3 a $9 \%$. O estudo evidenciou que $45 \%$ dos casos de câncer de mama foram relatados como localizados na mama, 20\% como regionais, e, aproximadamente, $10 \%$ dos casos foram diagnosticados com metástases a distância.

Este estudo temporal explica a diferença das taxas de casos avançados relatados por autores brasileiros na década de 1980 e início dos anos 1990, se comparados com os estudos mais recentes. Em decorrência do pequeno número de publicações analisando o comportamento temporal da neoplasia, o INCA estima ser a taxa de doença avançada próxima a $55 \%$, com pequena variação na última década ${ }^{1}$. No estudo brasileiro com maior casuística, publicado por Thuler e Mendonça ${ }^{6}$, em 2005, observou-se redução de 52 para $45 \%$ da taxa de casos avançados em diferentes centros de referência do Brasil. O Estado de São Paulo vem priorizando o acesso e resolução rápidos nos últimos quatro anos. Tal estratégia, reduziu o número de casos avançados (estádios 3 e 4) de 42 para cerca de $25 \%$ 2. 
Concluindo, o estudo tem grande importância no contexto da Saúde Pública no Brasil. Algumas limitações do trabalho decorrem da ausência de um registro nacional de câncer - em especial os de maior incidência. O estudo tem um diferencial por apresentar dados do Registro de Base Populacional da cidade de Goiânia operante há 20 anos. Representa, numa amostra populacional do Brasil, uma redução do número de casos avançados e um aumento das neoplasias in situ.

A avaliação periódica do estadiamento das neoplasias é fundamental para se avaliar a eficácia dos programas de detecção precoce, tratamento e mortalidade. Isso tem relevância não só no âmbito médico-científico, mas, principalmente, no redirecionamento de recursos aplicados em Saúde - seja ela pública ou privada.

O custo do tratamento médico é crescente em todo mundo, em especial em câncer de mama, o que nem sempre é revertido em redução mensurável da mortalidade. O estudo mostra a importância do rastreamento mamográfico, ainda que oportunístico, registrando também a necessidade da conscientização dos profissionais da área Saúde, para que haja direcionamento de recursos, visando aprimorar a coleta de dados, e para conhecer e monitorizar a incidência das neoplasias malignas e variáveis clínico-epidemiológicas, detectando mudanças no perfil de incidência temporal do câncer e a efetividade terapêutica.

\section{Referências}

1. Martins E, Freitas Junior R, Curado MP, Freitas NMA, Oliveira JC, Bandeira e Silva C. Evolução temporal dos estádios do câncer de mama ao diagnóstico em um registro de base populacional no Brasil Central. Rev Bras Ginecol Obstet. 2009;31 (5):219-23.

2. Fundação Oncocentro de São Paulo [Internet]. Dados de câncer: registro hospitalar de câncer, 2001-2008. São Paulo; 2008 [citado 2009 Abr 10]. Disponível em: http://www.fosp.saude.sp.gov.br/html/fr_dados.html

3. Smith RA, Cokkinides V, Brawley OW. Cancer screening in the United States, 2009: a review of current American Cancer Society guidelines and issues in cancer screening. CA Cancer J Clin. 2009;59(1):27-41.

4. Ministério da Saúde. Secretaria de Atenção à Saúde. Instituto Nacional de Câncer. Coordenação de Prevenção e Vigilância de Câncer. Estimativas 2008: incidência de câncer no Brasil. Rio de Janeiro: INCA; 2008.

5. Freitas-Junior R, Freitas NM, Curado MP, Martins E, Moreira MA, Silva CM. Variations in breast cancer incidence per decade of life (Goiânia, GO, Brazil): 16-year analysis. Cancer Causes Control. 2008;19(7):681-7.

6. Thuler LCS, Mendonça GA. Estadiamento inicial dos casos de câncer de mama e colo do útero em mulheres brasileiras. Rev Bras Ginecol Obstet. $2005 ; 27(11): 656-60$. 